\title{
MODELO PARA ANÁLISE ESTRATÉGICA DE INDÚSTRIAS BASEADAS EM CIÊNCIA
}

RESUMO

Este artigo apresenta um modelo para análise estratégica de indústrias em processo de capacitação tecnológica, baseadas em ciência e sediadas em países em desenvolvimento. 0 modelo é baseado em cinco elementos principais: regime tecnológico, estrutura mercadológica, processo organizacional, conhecimentos e recursos disponíveis, e papel do governo. Ele pode ser aplicado tanto no nível da indústria como no nível da empresa, e visa apoiar o alinhamento das estratégias de inovação à estratégia geral da empresa. Aplicado à indústria brasileira de vacinas, o modelo permitiu evidenciar limitações no regime tecnológico e nos processos organizacionais dos produtores brasileiros, bem como identificar oportunidades de crescimento mercadológico.

\section{Rosiceli Barreto Gonçalves Baetas}

UFRJ e Fundação Oswaldo Cruz

\section{José Vítor Bomtempo}

UFRJ

\section{Cristiane Quental}

Fundação Oswaldo Cruz

\begin{abstract}
This paper presents a strategic analysis model for science-based industries in process of innovation and technological development, operating in developing countries. The model is based on five main elements: technological regime; marketing structure; organizational process; knowledge and available resources, and governmental role. It can be applied in both industry and firm level, and aims to support the alignment of innovation strategies to general firm strategy. Applied to the Brazilian vaccines industry, the framework highlighted the Brazilian vaccine manufacturers' limitations in terms of technological regime and organizational processes, as well as the opportunities of market growth.
\end{abstract}

PALAVRAS-CHAVE Inovação, competitividade, estratégia, capacitação, indústria baseada em ciência. KEY WORDS Innovation, competitiveness, strategy, capability, science-based industry. 


\section{INTRODUÇÃO}

U ma questão fundamental para a gestão estratégica de empresas que atuam em setores industriais baseados em ciência em países em desenvolvimento é como se inserir competitivamente e de forma sustentável em um ambiente turbulento. Em um "setor baseado em ciência" o avanço tecnológico depende de conhecimentos, habilidades e técnicas provenientes da pesquisa científica (Pavitt, 1990), e a capacitação tecnológica e a inovação inovadora são fatores críticos de sucesso (Kim, 1997; Lall, 2000; Katz, 2000).

Este artigo apresenta uma estrutura analítica, denominada M odel o para Análise Estratégica de Indústrias Baseadas em Ciência de Países em Desenvolvimento ( $\mathrm{MAEI}$ ), que visa orientar a estratégia de organizações que estejam passando por processos de capacitação tecnológica. 0 modelo se baseia em cinco elementos: regime tecnológico, estrutura tecnológica, processos organizacionais, conhecimentos e recursos, e papel do governo.

N este artigo, a aplicação do M AEI éilustrada por uma análise da indústria brasileira de vacinas de uso humano. Ao mesmo tempo em que possui limitações estruturais, essa indústria está em posição favorável em termos de oportunidades, em função dos avanços da biotecnologia e da capacitação nacional.

0 artigo está organizado da seguinte forma: inicialmente, são discutidas as bases teóricas e conceituais da construção do modelo; em seguida, o modelo é apresentado e ilustrado pelo caso da indústria brasileira de vacinas; e, por fim, são apresentadas as conclusões e sugestões para outras aplicações do modelo.

\section{BASES DO MODELO}

O MAEI é baseado em quatro abordagens de análise estratégica: primeiro, nas capacitações dinâmicas de Teece, Pisano e Shuen (1997); segundo, na teoria de criação do conhecimento de Nonaka e Takeuchi (1995); terceiro, na trajetória reversa de Kim (1997); e quarto, no modelo de catching-up mercadológico e tecnológico de Lee e Lim (2001). Serão examinados a seguir os detal hes de cada um desses modelos.

\section{Capacitações dinâmicas}

U ma revisão da evolução dos modelos de anál ise estratégica, que têm como objetivo a formulação de estratégias para sustentar e aumentar a vantagem competitiva das empresas, aponta a existência de dois enfoques principais ao tema: o primeiro enfatiza o poder de mercado ou da indústria e suas interações estratégicas, e o segundo baseia-se nos recursos e na eficácia das empresas.

Em relação aos modelos que enfatizam o poder de mercado e interações estratégicas, destacam-se o modelo das cinco forças competitivas (Porter, 1980), os modelos que ampliam a análise dessas forças (Ghemawat, 1999) e o modelo da rede de valor (Brandenburger e Nalebuff, 1996).

As abordagens baseadas em recursos tiveram origem nos trabal hos de Penrose (1959), seguidos dos trabal hos de Nelson e Winter (1982) e Barney (1991). A partir da década de 1990, destacam-se abordagens baseadas em capacidades ou competências (Grant, 1991), que classificam os recursos como fontes de capacidades de uma empresa e capacidades como fontes de vantagens competitivas. Prahalad e Hamel (1990) conceituam competências essenciais como o conjunto de habilidades e tecnologias de difícil imitação que permitem à empresa oferecer um determinado benefício para os clientes e, eventualmente, entrar em outras indústrias.

Nessa mesma linha, a abordagem de Teece et al. (1997) tem como principal diferencial a ênfase na necessidade de adaptação contínua da empresa para garantir a inovação a longo prazo. Esses autores propõem que, em ambientes caracterizados por rápida mudança tecnológica, as capacitações dinâmicas são as fontes de criação, captação e manutenção de valor pelas empresas. Eles enfatizam três pontos que diferenciam sua abordagem das anteriores: a capacidade de renovar as competências, o papel-chave da administração estratégica, e a influência da trajetória na situação presente da empresa. Para os autores, as vantagens competitivas de uma empresa residem em seu processo organizacional, na posição de seus ativos específicos - tecnológicos, financeiros e mercadológicos - e na sua trajetória. 0 processo de aprendizado de uma empresa é dependente de sua trajetória, sendo que os caminhos são condicionados pelas competências acumuladas no desenvolvimento e exploração dos produtos.

Cohen e Levinthal (1990) propõem que as pré-condições necessárias para solucionar problemas no processo de inovação e no processo de aprendizagem são similares entre si. 0 aprendizado envolve o desenvolvimento de capacidade para assimilar o conhecimento existente, ao passo que a inovação requer a capacidade de criar novos conhecimentos. Em ambos os casos é a existência de conhecimento e habilidades relevantes que permite as associações e ligações necessárias para solucionar os problemas. 
Numa abordagem próxima à de recursos, Nelson e Winter (1982) e Pavitt (1990) enfatizam o papel da tecnologia. Pavitt (1990) explora a noção de trajetória tecnológica e o papel determinante das características do setor, e afirma que as diferenças e as similaridades entre os setores industriais estão relacionadas às fontes e direções das estratégias tecnológicas. Para o autor, as estratégias tecnológicas possíveis para uma empresa são determinadas pelas características do setor e sinal izam as potencialidades e limites que con duzem os esforços em uma certa direção. De acordo com Nelson e Winter (1982), o regime tecnológico podeser definido como o ambiente tecnológico onde a firma atua em determinado momento.

\section{Criação do conhecimento organizacional}

0 que há em comum nas abordagens apresentadas é que não exploram o processo de inovação em si. Para preencher essa lacuna, Nonaka e Takeuchi (1995) estudaram as interações que ocorrem nos planos individual e organizacional no processo de inovação. N onaka et al. (2000) propõem que a razão de ser da empresa é criar continuamente conhecimento, que é a mais importante fonte de vantagem competitiva.

Nonaka e Takeuchi (1995) argumentam que o processo de criação do conhecimento organizacional ocorre na interação, ao nível individual, entre os conhecimentos tácito e explícito. A inovação surge do processo de transformação, em duas dimensões: em uma dimensão, o conhecimento é criado e expandido por meio da interação social contínua e dinâmica entre os conhecimentos tácito e explícito; na outra dimensão, o conhecimento do indivíduo é transformado em conhecimento de grupo e conhecimento organizacional. A função da organização é prover as condições que auxiliem no processo de conversão do conhecimento e na formação das espirais do conhecimento.

\section{Modelos da trajetória reversa e de catching-up}

Grande parte da literatura sobre análise estratégica se destina aos países desenvolvidos. Em face das dificuldades de aplicação às empresas de países em desenvolvimento, diversas tentativas estão sendo desenvol vidas com o intuito de criar modelos que se adaptem às experiências de indústrias de países emergentes.

Diferentes autores vêm estudando as experiências de desenvolvimento tecnológico em países de industrialização recente, como as análises de experiências na Ásia de Pack (2000), Lall (2000) e Hobday (2000), e as análises de experiências de países da América Latina, inclusive do Brasil, de Katz (2000) e de Figueiredo (2003).
0 foco de interesse para o MAEI são as análises que tratam 0 aprendizado, a capacidade de absorção e a inovação como pontos centrais. Duas abordagens de experiências da Coréia do Sul possuem esse foco: o modelo da trajetória reversa de Kim (1997) e o modelo de catching-up mercadológico e tecnológico de Lee e Lim. (2001).

0 modelo dominante de análise do processo de evolução da inovação nos países desenvolvidos é o modelo da dinâmica da inovação de Utterback (1994), no qual a trajetória tecnológica das empresas segue três fases principais: fluida, transitória e específica. Na fase fluida ocorre um grande número de mudanças e o resultado é incerto. A fase transitória é caracterizada pela aceitação no mercado de uma inovação do produto. $\mathrm{N}$ a fase específica, 0 valor da relação qualidade-custo se torna a base da concorrência. Kim (1997) sugere que a trajetória das empresas de países em desenvolvimento segue as mesmas três fases, porém de forma inversa. Para ele, o desenvolvimento de capacitação tecnológica é visto como um processo de aprendizagem nos níveis individual e organizacional, e nas dimensões do conhecimento tácito e explícito. Um dos conceitos centrais da abordagem de Kim (1997) é que a capacidade de absorção de uma empresa é determinada pela base de conhecimento existente e pela intensidade de esforço comprometido. Para Kim, a capacitação tecnológica é um processo de aprendizagem que ocorre em todos os níveis da sociedade.

Lee e Lim (2001) também analisam alguns setores industriais da Coréia do Sul, buscando verificar as condições para a superação de etapas em direção à capacitação tecnológica. Para explicá-las, os autores construíram o modelo de catching-up mercadológico e tecnológico. Os autores defendem que o regime tecnológico é determinante nas atividades de inovação das empresas em processo de catching-up. Eles utilizam e apóiam o detal hamento de regime tecnológico feito por Malerba e Orsenigo (1993) - níveis de oportunidades e apropriabilidade, acúmulo de conhecimento ecomplexidade do conhecimento debase. Com isso, apresentam no modelo dois determinantes principais no processo de definição de produtos a desenvolver: o regime tecnológico e as fontes de vantagens competitivas. As políticas da empresa e o papel do governo complementam o modelo como determinantes do esforço de $P \& D$ que, por sua vez, afeta diretamente os resultados alcançados.

\section{MODELO INTEGRADO}

A idéia central que inspirou a proposição do $M A E I$ é que as qualidades dos quatro modelos estudados se reforçam 
quando os mesmos são considerados em conjunto. Além disso, considerou-se que as bases conceituais desses quatro modelos poderiam ser estruturadas em torno do que se denominou elementos do processo de capacitação tecnológica e inovadora em indústrias baseadas em ciência de países em desenvolvimento.

0 modelo das capacitações dinâmicas de Teece et al. (1997) trata de forma conjunta os ativos específicos da empresa e a posição mercadológica da empresa. Tal opção dificulta o processo de análise das capacitações, pois as análises de mercado e de ativos requerem ferramentas distintas. Dessa forma, na construção do M AEI o conceito "posição" de Teece et al. (1997) é subdividido.

Em sua teoria de criação do conhecimento, Nonaka e Takeuchi (1995) enfatizam aspectos relacionados às interações dos conhecimentos tácito e explícito, mas não incluem outros aspectos, como a trajetória e o regime tecnológico, também importantes no processo de capacitação tecnológica, que são abordados nos demais modelos.

O model o de Lee e Lim (2001), por outro lado, dá pouca importância à questão do processo organizacional , amplamente enfatizada nas outras abordagens. Além disso, esse model o objetiva ser uma ferramenta de apoio à administração estratégica, destinando-se apenas a explicar os três casos de catching-up estudados pelos autores.

Ambos os modelos baseados na experiência coreana (Kim, 1997; Lee e Lim, 2001) trazem reflexões sobre o processo de capacitação tecnológica. No entanto, exploram a idéia de um referencial dos países desenvolvidos no qual as empresas dos países em desenvolvimento se espelham. N esses modelos, o objetivo é analisar o processo de catching-up tomando-se como referência os produtos fabricados pelas empresas líderes. Entretanto, nem sempre é esse 0 objetivo dos produtores atuantes em países em desenvolvimento, pois muitos deles precisam achar nichos para atuar, visto que, em geral, não possuem condições de competir diretamente com os líderes.

\section{0 modelo proposto: MAEI}

O MAEI aplica-se tanto à anál ise de indústrias quanto ao apoio à gestão estratégica de uma organização em particular. Ele permite a identificação de limitações e oportunidades que podem ser exploradas no nível da indústria e no da empresa ou agente produtor. No primeiro caso, visa fornecer um retrato da situação geral da indústria e pode ser usado, por exemplo, como apoio à definição de políticas setoriais. Quanto à sua aplicação no nível da organização, o MAEI ajuda no entendimento das potencialidades da organização frente ao ambiente, na identificação de escolhas possíveis e na definição de estratégias, sendo ainda útil para o al inhamento das estratégias de inovação à estratégia geral da organização.

No modelo considera-se que um produto é fruto de inovação quando é novo para a organização em análise, mesmo que não o seja para os competidores (Mytelka, 1999). Em função do estágio de desenvolvimento tecnológico da organização, será necessário desenvolver capacidade para assimilar o conhecimento existente (Cohen e Levintal, 1990). Assim, em muitos casos deverá se percorrer uma trajetória reversa à de Kim (1997): no primeiro estágio, a organização adquire tecnologias em fase específica (ou madura); no segundo estágio (imitação criativa), repete o processo com tecnologias ainda em transição; e no terceiro estágio, é capaz de gerar tecnologias emergentes, em fase fluida.

No MAEI, considera-se como complementares as abordagens baseadas no mercado (Porter, 1980; Ghemawat, 1999; Bradenburger e Nalebuff, 1996) e aquelas baseadas em recursos (Penrose, 1959; N el son e Winter, 1982; Barney, 1991; Grant, 1991; Prahalad e Hamel, 1990) e no ambiente tecnológico (Pavitt, 1990; N elson e Winter, 1982; Lee e Lim, 2001). A identificação de oportunidades mercadológicas e tecnológicas deve ser feita à luz das capacitações dinâmicas (Teece et al., 1997), levando-se em conta os ambientes mercadológico e tecnológico nos quais a organização atua e o papel do governo (Lee e Lim, 2001). O processo organizacional - adaptado de Teece et al. (1997) e N onaka e Takeuchi (1995) - deve oferecer condições de capacitação para a organização aprender e inovar. 0 desenvolvimento de capacitação tecnológica e inovadora é visto principal mente como um processo de aprendizagem.

0 modelo (Figura 1) contém cinco el ementos principais relacionados às escolhas das estratégias para o desenvolvimento de capacitação tecnológica e inovadora: o primeiro refere-se ao regime tecnológico e à posição relativa da empresa; o segundo refere-se à estrutura mercadológica e à posição relativa da empresa; o terceiro relaciona-se ao processo organizacional; o quarto refere-se aos conhecimentos e recursos disponíveis; e o quinto refere-se ao papel desempenhado pelo governo. Cada um desses elementos é descrito a seguir.

As análises do regime tecnológico e da estrutura mercadológica devem ser realizadas no nível internacional, exceto quando houver limites relevantes do ambiente competitivo da indústria dados por condições locais. A análise do regime tecnológico tem como objetivo apontar as oportunidades de desenvolvimento ou incorporação de novos produtos à linha atual da empresa, em face 
das características específicas segundo as quais as atividades de inovação são organizadas. Essa análise pode explorar tanto o regime tecnológico da indústria em que a organização atua como o regime tecnológico de novos usos potenciais para as competências e ativos existentes.

A análise dos conhecimentos e recursos disponíveis deve apontar as vantagens e desvantagens das escolhas estratégicas possíveis. Essa análise pode compreender duas abordagens: na primeira, pode-se focar a indústria em que a empresa atua, e na segunda, a avaliação deve se debruçar sobre novos usos para os ativos e competências existentes. Essa análise pode ser ampliada com o intuito de explorar as necessidades de aprimoramento ou mesmo desenvolvimento de novos conhecimentos e recursos para fazer frente às oportunidades identificadas.

As decisões estratégicas de aprendizado e inovação estão relacionadas às possibilidades e às decisões de explorar as oportunidades. Essas oportunidades podem ter uma natureza de curto ou longo prazo e, em ambas as condições, podem estar relacionadas ou não à indústria em que a organização atua e aos ativos e competências de que a organização dispõe (Loewe et al., 2001). As estratégias gerais da empresa devem orientar essas decisões, e o processo organizacional deve ser analisado levando-se em conta se está estruturado para favorecer a identificação de oportunidades, a inovação e o aprendizado, e de que modo esse processo pode ser melhorado em função das decisões estratégicas.

As estratégias de inovação relacionadas à indústria em que a organização atua são aplicadas quando existe potencial de crescimento na indústria ou dentro dela, ou ainda perspectiva de oportunidades futuras, originadas por um novo ciclo de inovação. Para as oportunidades de curto prazo, a organização deve promover inovações e aprendizados rápidos, com vistas a gerar melhorias e mudanças rápidas (Loewe et al., 2001). As estratégicas de inovação relacionadas a novos usos para os ativos e competências se aplicam em duas situações principais: quando a empresa percebe a necessidade de buscar novos usos para os ativos e competências ou quando são identificadas novas oportunidades para estes.

Por fim, deve ser analisado o papel do governo e as suas interferências no regime tecnológico, a estrutura mercadológica, o processo organizacional, e os conhecimentos e recursos disponíveis. Devem-se focar as políticas industriais, de ciência, tecnologia e inovação e setoriais que afetam as estratégias da empresa e, principalmente, as estratégias de inovação.

\section{Regime tecnológico}

0 regime tecnológico reflete como as atividades de inovação de um determinado setor são organizadas, e como

Figura 1 - Modelo para Análise Estratégica de Indústrias baseadas em Ciência de Países em Desenvolvimento - MAEI.

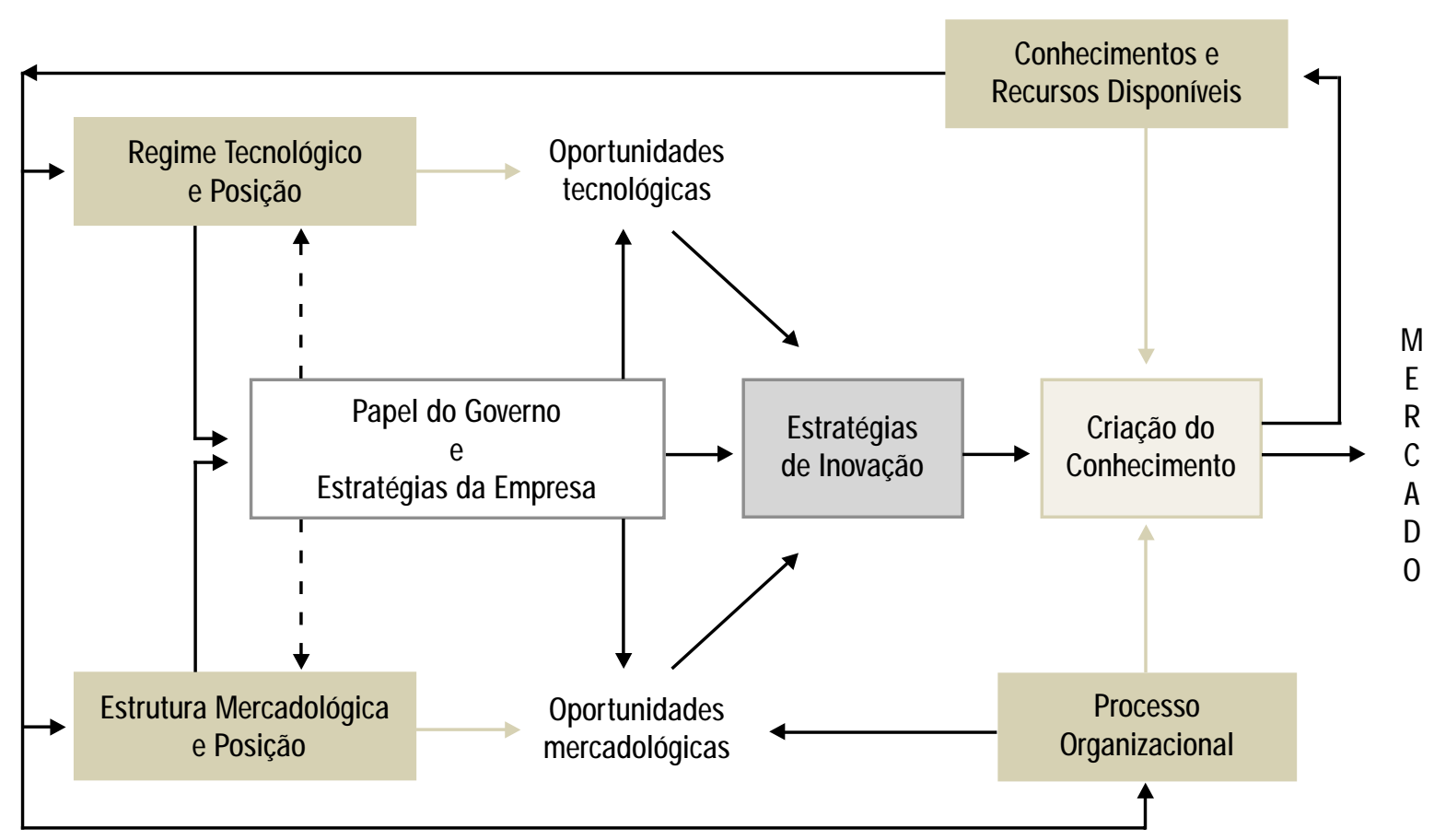


elas afetam diretamente as oportunidades tecnológicas e as oportunidades para o desenvolvimento de produtos. 0 regime tecnológico é definido neste artigo como a combinação dos seguintes fatores: fluidez da trajetória tecnológica, freqüência da inovação, fonte da inovação, possibilidades de acesso à tecnologia ou condições de apropriação da inovação, e possibilidades de acesso aos ativos complementares.

A trajetória tecnológica pode ser mais ou menos previsível. Define-se como fluidez da trajetória tecnológica o grau de acumulação do desenvolvimento tecnológico e a previsibilidade da direção desse desenvolvimento. Pode-se utilizar aqui o conceito das fases de Utterback (1994) e classificar a trajetória como fluida, transitória ou específica. Como afirmam Lee e Lim (2001), a freqüência da inovação também éimportante, pois uma al ta ou baixa incidência de inovações num período curto de tempo afeta as oportunidades da empresa.

Outros fatores de grande importância são as fontes da inovação e as possibilidades de acesso à tecnologia. Para a verificação das possibilidades de acesso à tecnologia já existente devem ser anal isadas as fontes de aprendizado tecnológico (Kim, 1997; Lee e Lim, 2001). No caso de inovações recentes, as análises se voltam para a capacidade de aprendizado e absorção. Devem também ser analisadas as condições de apropriação dessas inovações (Teece, 1986). Essas condições estão relacionadas à capacidade de proteger a inovação e ao acesso aos ativos complementares. Tal análise pode ser feita tanto do ponto de vista de se apropriar de inovações externas quanto para proteger a própria inovação.

Algumas oportunidades de inovação exigem ativos especializados que o inovador não detém, mas que precisam estar disponíveis para viabilizar a venda do produto: são os ativos complementares. As al ternativas são integrar os ativos ou realizar acordos com outras empresas. As possibilidades de acesso a tais ativos também devem ser analisadas (Teece, 1986).

\section{APLICAÇÃO DO MODELO}

O MAEI foi utilizado na análise da indústria de vacinas, tendo em vista a sensibilidade desse setor às inovações tecnológicas e sua rel evância no quadro nacional do sistema de saúde. A seguir será detal hado cada um dos cinco elementos do modelo.

\section{Regime tecnológico da indústria brasileira de vacinas}

A trajetória tecnológica na indústria de vacinas se carac- teriza por duas grandes fases, divididas nos períodos anterior e posterior ao advento da biotecnologia moderna. Em cada uma dessas fases observam-se ciclos distintos de inovação (Galambos e Sewell, 1995). De um primeiro ciclo, que compreende um período de ênfase bacteriológica, virológica e de nova bacteriologia de cápsulas polissacarídicas, surgiram as chamadas vacinas de primeira geração, que são as vacinas inativadas e atenuadas. As vacinas de segunda geração são mais modernas, utilizando novas técnicas da biotecnologia moderna, decorrentes do ciclo do DNA recombinante, dos estudos moleculares da genética e do ciclo da tecnologia de conjugação. As novas abordagens no desenvolvimento de vacinas têm evoluído na direção do desenvolvimento das chamadas vacinas de terceira geração, como as vacinas de DNA (The Jordan Report, 2002).

Do ponto de vista do conceito de fluidez da trajetória tecnológica, observa-se no setor de vacinas a convivência das fases fluida, transitória e específica. De um lado, têm-se inovações incrementais, como as vacinas combinadas, que são novas apresentações das vacinas existentes, o que é característico de fase específica; de outro, observam-se características de fase transitória, como as inúmeras vacinas em estudos clínicos no mundo provenientes do ciclo da DNA recombinante e estudos moleculares da genética. A fase fluida pode ser ilustrada pelas vacinas de terceira geração em desenvolvimento, como as vacinas contra AIDS e Malária, para as quais não foi possível utilizar as abordagens das vacinas de primeira geração.

Q uanto à freqüência de inovação, a história do desenvolvimento de vacinas tem mais de 200 anos, mas até a década de 1980 existiam aproximadamente 20 vacinas licenciadas nos Estados Unidos. Entretanto, a partir dessa década os avanços na biotecnologia e na imunologia permitiram o surgimento de uma nova geração de vacinas, e, desde então, foram licenciadas mais de 20 novas vacinas de uso humano (The Jordan Report, 2002).

As fontes de inovação da moderna biotecnologia têm sido as universidades, os centros de pesquisa e as novas empresas de base biotecnológica (NEBs). A maioria das NEBs nasceu a partir de 1980 em ambientes acadêmicos e são caracterizadas como empresas inovadoras, baseadas em pesquisa e detentoras de tecnologia patenteada. A competência estratégica dessas empresas fundamentase nas pesquisas científicas, enquanto seus pontos fracos são os ativos complementares para a produção em ampla escala, marketing, capacitação gerencial para a real ização de estudos clínicos e atendimento das exigências regulatórias, que são por sua vez competências abundan- 
tes nas líderes farmacêuticas. As NEBs foram e ainda são alvo de interesse estratégico das empresas líderes, motivadas pela necessidade de capacitação na biotecnologia moderna.

Uma análise da trajetória tecnológica dos produtores dos países em desenvolvimento mostra uma história de sucessivos acordos de transferência de tecnologia com os países desenvolvidos, como o Brasil. Por outro lado, observam-se também trajetórias de inovação a partir da biotecnologia moderna, como ocorre com al guns produtores em Cuba.

\section{Estrutura mercadológica}

A análise da estrutura mercadológica fornece indicações das oportunidades mercadológicas para a empresa. 0 objetivo é facilitar a identificação das vantagens competitivas que a empresa vem construindo e suas potencialidades à luz da dinâmica da estrutura industrial.

A estrutura industrial é o ambiente dinâmico onde se relacionam os competidores, clientes, fornecedores, entrantes potenciais, substitutos, complementadores e demais participantes rel evantes (Porter, 1980; Ghemawat, 1999). Como as relações dos participantes da indústria tendem a mudar com o tempo, o mapeamento das relações futuras fornece informações úteis (Ghemawat, 1999).

Para a identificação dos determinantes das vantagens competitivas é conveniente fazer uma análise dos direcionadores de custo e da diferenciação. 0 objetivo é identificar as oportunidades para aumentar o valor apropriado pela empresa com as atividades desempenhadas (Porter, 1985). Também devem ser anal isados os comprometimentos existentes: as decisões que envolvem grandes mudanças na modalidade de recursos e, portanto, possuem características de irreversibilidade (Ghemawat, 1999).

\section{A estrutura mercadológica da indústria de vacinas}

0 setor de vacinas passou por um processo de consolidação nas duas últimas décadas, fazendo com que hoje possua características oligopolistas: quatro empresas detêm mais de $80 \%$ do faturamento do setor. A estratégia das empresas baseia-se no lançamento contínuo de produtos protegidos por patentes e de preço mais elevado, com a expectativa de aumentar as vendas e os lucros anuais (Merrill Lynch, 2003).

Um outro aspecto importante para se compreender 0 mercado de vacinas é o papel de organismos internacionais, como a Organização Mundial de Saúde (OMS) e o Fundo das Nações Unidas para Crianças (UNICEF). Esses organismos possuem o papel de estimuladores da produção de vacinas em países em desenvolvimento.
Atualmente, as novas vacinas estão sendo introduzidas nos países em desenvolvimento principalmente por meio de acordos de transferência de tecnologia e licenças de exploração de patentes ou joint-ventures. Em alguns casos, como no Brasil, a negociação de tais acordos é mediada pelo poder de barganha dos governos, que detêm o mercado público. É essa lógica que tem norteado os acordos recentes no Brasil entre os produtores públicos locais e as empresas líderes.

Uma das características do mercado de vacinas é a sua segmentação, fazendo com que os preços e tipos de vacinas variem conforme o segmento. Nos países em desenvolvimento, há uma diferença relevante entre os preços e tipos de vacinas dos setores governamental e privado, fazendo com que surjam oportunidades para os produtores locais (Kremer, 2000).

Em relação à pesquisa e ao desenvolvimento das vacinas, ambos são realizados basicamente nos países desenvolvidos. 0 foco das pesquisas está vol tado às doenças que se manifestam mais comumente em tais países, fazendo com que as pesquisas das doenças mais comuns em países em desenvolvimento, como a dengue, a febre amarela a leishmaniose, não constituam prioridade. Por outro lado, de certa forma esse desinteresse representa uma oportunidade para os produtores dos países em desenvolvimento.

As vacinas possuem uma característica particular no que diz respeito a insumos. Ao se desenvolver, testar clinicamente e aprovar uma vacina, o insumo principal é o resultado do seu próprio desenvolvimento: o lote-semente primário, que dá origem aos lotes a serem utilizados nos testes clínicos. A estratégia de cópia é dificultada para os países em desenvolvimento, pois, para provar que um produto copiado é igual mente seguro e eficaz, é necessário realizar novos testes clínicos. Além disso, existem ameaças de produtos substitutos, como novas vacinas combinadas em substituição às vacinas monovalentes; novas abordagens para a aplicação das vacinas (aerossol nasal e adesivos) e vacinas comestíveis (produtos geneticamente modificados para conter antígenos contra determinadas doenças).

Os fabricantes de vacinas podem ser classificados em quatro grupos estratégicos: a) multinacionais com atuação global; b) novas empresas de base biotecnológica NEBs; c) empresas seguidoras; e d) empresas focalizadas. No primeiro grupo se encontram as divisões de vacinas de grandes empresas farmacêuticas baseadas em pesquisa. No das NEBs, poucas empresas evoluíram até a etapa de comercialização de vacinas. No grupo de seguidoras, encontram-se as empresas, institutos públicos e institutos privados que atuam principalmente no mercado domésti- 
co ou como fornecedores da OMS e do UNICEF. Países com a Índia, a China, a Indonésia e o Brasil possuem produtores com essa característica. 0 último grupo é composto de empresas, institutos públicos e institutos privados inovadores, com foco restrito de atuação. Os produtores cubanos também se encontram nessa categoria.

No Brasil, o mercado de vacinas é afetado de forma considerável pelas motivações de saúde pública, porém limitadas por aspectos políticos e econômicos. 0 governo brasileiro coordena a aquisição e distribuição gratuita de vacinas segundo um calendário estabelecido, cuja decisão de inclusão ou não de determinadas vacinas depende de critérios tanto epidemiológicos quanto econômicos. Observa-se uma contínua pressão pela introdução de novas vacinas no calendário oficial. Temporão (2002) observa que, apesar de a oferta nacional ser crescente, ainda há dependência externa, sobretudo das novas vacinas. A demanda da área privada é crescente e total mente atendida por importações.

O Brasil conta com dois produtores públicos: o Instituto de Tecnologia em Imunobiológicos de $M$ anguinhos (Bio-Manguinhos), da Fundação Oswaldo Cruz, vinculado ao M inistério da Saúde, e o Instituto Butantan, subordinado ao governo do Estado de São Paulo. As suas linhas de vacinas são complementares e estão incorporando novos produtos, principalmente por meio de acordos de transferência de tecnologia com empresas multinacionais. Em outros países, como na Índia, China, Indonésia e Cuba, existem muitas empresas e instituições de porte pequeno a médio.

Diante desse cenário, é possível identificar algumas arenas competitivas e segmentos com necessidades diferenciadas que podem ser explorados pelos países em desenvolvimento, com variações em opções estratégicas. Por exemplo, para a estratégia de inovação com foco na indústria, existem opções de baixo custo com atuação global ou local, o que exige eficácia, para garantir custo competitivo, e estratégia, para focal izar segmentos de mercado. Entretanto, existem também restrições de natureza legal - patentes, estudos clínicos - e tecnológica à cópia de vacinas já existentes, bem como gargal os de natureza científica, como os enfrentadas para o desenvolvimento de vacinas para determinadas doenças.

\section{0 processo organizacional}

0 exame do regime tecnológico e da estrutura mercadológica fornece as peças-chave para o processo de formulação ou construção da estratégia. 0 processo organizacional aborda o "como fazer", fornecendo as condições de capacitação para a implementação ou o desenvolvimento da estratégia.
0 processo organizacional é o modo como a empresa se estrutura, e em que grau sua estrutura favorece a inovação, sendo o aprendizado um dos principais fatores (Kim, 1997). Quanto mais a empresa for organizada para o aprendizado, mais chances terá de se capacitar em termos de tecnologia e inovação. Outro fator é a habilidade de se reconfigurar, ou seja, de realizar as transformações necessárias exigidas num ambiente de rápidas mudanças (Teece et al., 1997).

Dessaforma, o processo organizacional compreendecompetências organizacionais, relacionais e técnicas (François et al., 1999). As competências organizacionais estão ligadas à capacidade da empresa de criar novos conhecimentos na gestão dos recursos humanos, tais como a capacidade de favorecer e canalizar a criatividade, e a capacidade de se transformar ede proteger a propriedade intelectual. Ascompetências relacionais dizem respeito à capacidade de integrar e coordenar atividades internas e externas, estabelecer alianças, integrar novas tecnologias, coordenar novas atividades, acompanhar, prever e agir sobre a evolução do mercado. A competência técnica está ligada à capacidade da empresa de detectar as necessidades ou possibilidades de melhorias de produtos, processos e organizacionais, bem como de introduzir inovações rapidamente.

Todas essas competências são classificadas segundo os elementos do MAEI, buscando verificar se favorecem os produtores quanto ao posicionamento tecnológico e mercadológico, e se favorecem a utilização dos recursos. 0 que deve ser anal isado, portanto, são principalmente as competências que favorecem o aprendizado contínuo e a inovação desenvolvidos pela firma.

\section{0 processo organizacional e a indústria de vacinas}

A maior parte das informações apresentadas a seguir é baseada no estudo de Bomtempo e Baetas (2004) acerca dos produtores brasilei ros. Tais informações levam a crer que há possibilidades para estruturar essas instituições com a finalidade de aprendizado e inovação. Uma das questões discutidas por especial istas do setor é se o ponto central das limitações dos produtores brasileiros é decorrente de sua natureza jurídica e organizacional de instituição pública. Não há consenso, mas certamente a falta de flexibilidade administrativa é um complicador. U ma alternativa prática para incentivar a flexibilidade foi desenvolvida pelos institutos Bio-Manguinhos e Butantan, com a criação de fundações de apoio.

As instituições brasileiras produtoras de vacinas são aval iadas por Bomtempo e Baetas segundo 12 dimensões organizacionais consideradas fundamentais para o favorecimento do aprendizado contínuo e da inovação: 1) 
realização de inovação; 2) posicionamento tecnológico; 3) monitoramento das tecnologias; 4) capacidade de apropriação das tecnologias; 5) conhecimento, previsão e ação sobre a evolução dos mercados; 6) recursos físicos e financeiros; 7) capacidade de gerir e defender a propriedade intelectual; 8) inserção da inovação e do aprendizado na estratégia de conjunto da organização; 9) capacidade de organizar e dirigir a produção, e assimilação e difusão interna de conhecimento; 10) gestão de RH na perspectiva de aprendizado e inovação; 11) financiamento das inovações e assimilação de tecnologias; e 12) cooperação.

No presente artigo, toma-se como premissa que as dimensões identificadas como favorecedoras de um mel hor posicionamento tecnológico dos produtores de vacinas são o monitoramento das tecnologias, a capacidade de apropriação das tecnologias e a capacidade de gerir e defender a propriedade intelectual. É importante ressaltar que a dimensão capacidade de apropriação de tecnologias também é anal isada no item regime tecnológico.

Bomtempo e Baetas apontam para a necessidade de se aperfeiçoarem os mecanismos de monitoramento das tecnologias existentes, como o uso de fontes externas para o monitoramento das tecnologias de concorrentes e das tecnologias de futuro, além de procedimentos formais para a análise de publicações dos profissionais dos concorrentes e as patentes destes.

Quanto à capacidade de apropriação das tecnologias, observa-se a necessidade de se buscarem sistematicamente novos desenvolvimentos e habilidades para imitar e copiar. Os dois principais produtores brasileiros possuem grupos próprios de pesquisa e um razoável acesso às fontes de tecnologia de fase específica, pois contam com acordos de transferência de tecnologia com empresas estrangeiras. Porém, a subcontratação de $P \& D$ é limitada, sobretudo no exterior. Há espaço para um fortalecimento das interações pesquisa-produção e as equipes de $P \& D$ ainda carecem de engenheiros dedicados às especificações de projetos. Vem crescendo a atenção das instituições quanto à capacidade de gerir e defender a propriedade intelectual, mas não existem procedimentos formais relacionados.

A dimensão organizacional identificada como favorecedora de um melhor posicionamento mercadológico referese ao conhecimento, previsão e ação sobre a evolução dos mercados. A principal limitação dos produtores brasileiros na exploração e identificação de novas oportunidades é o foco no mercado interno.

Nos processos organizacionais que favorecem uma mel hor utilização dos recursos, são identificadas as seguintes dimensões: inserção do aprendizado e inovação na es- tratégia de conjunto da organização; gestão de recursos humanos na perspectiva de aprendizado e inovação; capacidade de organizar e dirigir a produção, e assimilação e difusão interna de conhecimento; capacidade de financiar as assimilações e inovações de tecnologias; e capacidade de cooperação. Quanto às três primeiras dimensões, observou-se, neste estudo, necessidade de mel horias. Quanto à capacidade de financiamento, os produtores brasileiros têm conseguido financiar as aquisições de tecnologias; entretanto, os gastos em $P \& D$, financiados principalmente pelos governos federal e estadual, são considerados insuficientes.

Quanto à cooperação entre os grupos de $P \& D$ de vacinas no país, identificou-se a necessidade de coordenação das pesquisas. A cooperação externa é limitada. Em Cuba existe uma estreita colaboração entre todos os institutos envolvidos com pesquisa, desenvolvimento, estudos clínicos e produção de vacinas, além de uma coordenação central. Tais características têm sido apontadas como pontos fortes do desenvolvimento biotecnológico cubano.

\section{Conhecimentos e recursos disponíveis}

0 processo organizacional diz respeito ao "como" a empresa funciona, enquanto os conhecimentos e recursos disponíveis examinam "o que" a empresa tem disponível para funcionar e, principal mente, se está capacitada para fazer frente às oportunidades. A análise aqui se volta para os recursos físicos, humanos e tecnológicos. 0 objetivo é avaliar se a empresa dispõe de recursos e conhecimentos para realizar trabal hos de pesquisa e desenvolvimento, para financiar uma inovação, se dispõe de tecnologias-chave, de capacidade produtiva, de logística e de marketing. Além disso, é importante verificar se as competências existentes na empresa podem ser aplicadas a outras indústrias, caso existam competências essenciais que extrapolem as fronteiras setoriais (Hamel e Prahalad, 1994).

A existência de infra-estrutura é fundamental, tanto para produção quanto para desenvolvimento tecnológico, com padrões internacionais de boas práticas de fabricação. No Brasil, os produtores ainda não contam com a infra-estrutura dedicada exclusivamente à produção de lotes para estudos clínicos, mas existem projetos de construção em andamento. 0 volume de recursos gastos em pesquisa e desenvolvimento pelos produtores e demais grupos de pesquisa é considerado muito limitado. Quanto aos recursos humanos, é importante mencionar a necessidade de contar com uma formação multidisciplinar e ao nível de doutorado nas equipes de $P \& D$.

Observa-se que existem plataformas biotecnológicas comuns que podem ser aplicadas a vários setores da bio- 
tecnologia, como reativos para diagnóstico, biofármacos e derivados de plasma. A maioria das empresas e instituições analisadas atua em alguma dessas outras áreas. Entretanto, evidencia-se que os produtores brasileiros são focados predominantemente em vacinas, enquanto outros, como os cubanos, por exemplo, são bem mais diversificados em sua base biotecnológica.

\section{Papel do governo}

Políticas gerais para promover a indústria, a educação e o comércio certamente afetam os componentes regime tecnológico, estrutura mercadológica, processo organizacional, e conhecimentos e recursos disponíveis, requerendo análises complexas. Para fins deste trabalho, propõe-se restringir a análise às políticas de ciência, tecnologia e inovação $(C \& T \& I)$, com destaque para os aspectos relacionados ao fomento. A questão central é avaliar se tais políticas estão ajustadas ao estágio de desenvolvimento da indústria e do sistema que sustenta 0 aprendizado e a inovação na indústria.

Assim, uma primeira questão a ser relevada é se há um reconhecimento explícito do Estado de que $C \& T \& I$ são ferramentas do desenvolvimento industrial e, em caso afirmativo, qual o papel na indústria em análise nesta pesquisa. Segundo Dogson (2000), as políticas de $C \& T \&$ I se sobrepõem, mas para facilitar as análises podem ser subdivididas da seguinte forma: científicas, que dizem respeito à promoção da ciência em universidades e laboratórios de pesquisa; tecnológicas, relacionadas ao desenvolvimento de tecnologias genéricas, como tecnologia da informação, biotecnologia, eletrônica e aeroespacial; e de inovação, que são políticas de incentivo à acumulação, à difusão e à criação de novos produtos, processos e serviços pelas empresas.

A atuação do governo no incentivo às indústrias baseadas em ciência é fundamental e, especificamente na questão das vacinas, sua rel evância ganha uma dimensão maior, em função do impacto sobre a saúde pública. Como já assinalado, o governo nos países desenvolvidos étambém comprador. No Brasil, o governo é comprador e produtor. As duas principais instituições produtoras são públicas, atendendo principalmente à demanda pública federal .

As políticas governamentais para o setor de vacinas têm tido como objetivo, em vários países em desenvolvimento, garantir o suprimento de vacinas a baixo custo. N esse contexto se buscou fortalecer a produção local, como ocorreu no Brasil. A estratégia local está voltada para atender as necessidades do mercado interno, a partir das demandas do Ministério da Saúde. De forma alternativa, Cuba, face à sua limitada dimensão populacional, focalizou a biotecnologia e as vacinas como uma oportunidade de inserção competitiva e, dessa forma, vem incentivando a inovação e focando o mercado internacional.

No Brasil, observa-se nos últimos anos que a ênfase na eficiência orçamentária do governo acarretou uma pressão nos produtores públicos para garantir um baixo preço. Essa pressão, importante em vários aspectos, ensejou, porém, uma visão de curto prazo, visto que não foi acompanhada de uma política de desenvolvimento tecnológico e de inovação. As políticas científicas brasileiras estão refletidas no número de grupos de pesquisa - aproximadamente 60 -, localizados nas universidades e centros de pesquisa, que atuam no estudo de vacinas no país. Entretanto, há um reconhecimento geral da fal ta de recursos para manter os grupos ativos e da falta de articulação com o setor produtivo.

Especialistas e estudiosos do setor, como Homma et al. (2003), apontam o desenvolvimento tecnológico como elo deficiente na inovação tecnológica de vacinas no Brasil. Gadel ha (2003) chama a aten ção para os constrangimentos estruturais do setor e Temporão (2002; 2003) analisa o papel do Programa Nacional de Imunizações no desenvolvimento e na conformação do setor. Sob o ponto de vista do papel do governo, uma análise desses estudos mostra que uma questão comum é a fal ta de uma política tecnológica e de inovação para o setor.

\section{CONCLUSÃO}

As considerações que seguem abordam dois aspectos principais: o primeiro relacionado ao modelo proposto e o segundo, aos resultados da análise da indústria brasileira de vacinas.

A discussão da pertinência de utilização dos modelos selecionados de anál ise estratégica - baseados em conhecimento e inovação - em empresas sediadas em países em desenvolvimento, atuantes em setores baseados em ciência, em processo de capacitação tecnológica e inovadora apontou que os modelos de países desenvolvidos analisados, apesar de oferecerem amplas contribuições para reflexão, possuem limitações para os países em desenvolvimento. Adicionalmente, as análises de experiências de inserção competitiva de empresas situadas fora do eixo EUA-Europa-Japão, especial mente a Coréia do Sul, oferecem elementos de reflexão úteis para empresas que devem estar voltadas para a inovação.

O MAEI constitui uma ferramenta de avaliação dos agentes determinantes do contexto operacional das empresas baseadas em ciência, pois permite conjugar as abordagens de diferentes fatores e eventos. A análise de cada 
um dos cinco el ementos que compõem o modelo permite a identificação de limitações e oportunidades que podem ser exploradas tanto no nível da empresa ou agente produtor quanto no nível da indústria.

Portanto, o model o se configura como um instrumento para a análise da indústria, podendo orientar o processo de capacitação tecnológica e inovadora das empresas e do setor em questão. O MAEI pode ajudar a empresa no entendimento das suas potencial idades frente ao ambiente, na identificação de escol has possíveis e na definição de estratégias de aprendizado e inovação. Além disso, parece promissora a aplicação do modelo em segmentos industriais não necessariamente baseados em ciência.

No caso específico do setor de vacinas, o MAEI mostrou-se adequado para o entendimento desse setor. $\mathrm{Ob}$ serva-se que a indústria de vacinas brasileira, foco da análise, conta com uma base produtiva estabelecida e com oportunidades de crescimento mercadológico, porém possui limitações em seu posicionamento no regime tecnológico e em seu processo organizacional. Quanto ao regime tecnológico, verificam-se dificuldades para conciliar a convivência de um ambiente afeito tanto à fase específica, em face da necessidade de atuação em produtos maduros, como à fase fluida, frente às novas abordagens de desenvolvimento de vacinas. No processo organizacional, observam-se dificuldades relacionadas, sobretudo, à gestão da inovação. Como o papel do governo é importante, dada sua natureza de comprador e produtor do governo brasileiro, observa-se a necessidade de uma melhor integração das ações das esferas governamentais responsáveis pela saúde, $C \& T \& I$ e indústria, com vistas à promoção do setor.

Os produtores brasileiros se defrontam com possibilidades de decisões estratégicas relacionadas tanto à exploração de oportunidades na indústria - de curto e de longo prazo -, quanto à exploração dos conhecimentos e recursos existentes. Existem oportunidades advindas dos novos ciclos de inovação na indústria, de ampliação de mercado, de demandas não atendidas e de diversificação na biotecnologia aplicada à saúde. As oportunidades existem, e as decisões políticas que precisam ser tomadas podem dispor de um instrumento gerencial que auxilia a determinação dos objetivos estratégicos e a formulação de estratégias de negócio eficazes para empresas baseadas em ciência.

\section{NOTA}

O autor José Vítor Bomtempo registra seu agradecimento ao apoio do CN Pq e Faperj.

\section{REFERÊNCIAS BIBLIOGRÁFICAS}

BARNEY, J. Firm resources and sustained competitive advantage. Journal of Management, v. 11, n. 4, p. 99-120, 1991.

BOMTEMPO, J. V.; BAETAS, R. Potencialidade para o desenvolvimento de vacinas no Brasil. Vacinas, soros e imunização (no prelo). Rio de Janeiro: Fundação O swaldo Cruz, 2004.

BRADENBURGER, A.; NALEBUFF, B. Co-opetition. New York: Currency Doubleday, 1996.

COHEN, W.; LEVINTHAL, D. Absorptive capacity: a new perspective on learning and innovation. Administrative Science Quaterly. v. 35, n. 1, p. 128-152, 1990.

DOGSON, M. Policies for science, technology, and innovation in Asian newly industrializing economies. Technology, Innvation and Learning. Cambridge (UK): Cambridge University Press, 2000.

FIGUEIREDO, P. Learning, capability accumulation and firms differences: evidence from latecomer steel. Industrial Corporate Change, v. 12, n. 3, p. 607-643, 2003.

FRAN ÇOIS, J. et al. D écrire les compétences pour l'ínnovation. In: Foray D. et Mairesse J. (org.). Paris: Editions EHESS, p. 283-303, 1999.

GADELHA, C; AZEVEDO, N. Inovação em vacinas no Brasil. História, Ciências, Saúde - Manguinhos, v. 10, Suplemento 2, Rio de Janeiro, Fundação Oswaldo Cruz, 2003.

GALAMBOS, L.; SEW ELL, J. N etworks of Innovation - Vaccine D evelopment at Merck, Sharp \& Dohme and Mulford. Cambridge (UK): Cambridge University Press, 1995.

GHEM AWAT, P. Strategy and the Business Landscape. Reading (MA): Addison Wesley, 1999.

GRANT, R. The Resourse-based theory of competitive advantage: implications for strategy formulation. California M anagement Review, v. 33, n. 3, p. 114-135, 1991.

HAMEL G.; PRAHALAD C. Competing for the Future. Cambridge (MA): Harvard Business School Press, 1994.

HOBDAY, M. East versus Southeast Asian innnovation systems. Technology, Learning \& Innovation. Cambridghe(UK): Cambridge University Press, 2000.

HOMMA, A. et al. Desenvolvimento tecnológico: elo deficiente na inovação tecnológica de vacinas. História, Ciências, Saúde-Manguinhos, v. 10, Suplemento 2, Rio de Janeiro, Fundação Oswaldo Cruz, 2003.

KATZ, J. The Dynamics of Technological Learning during the ImportSubstitution Period and Recent Structural Changes in Industrial Sector of Argentina, Brazil and M éxico. Technology, Learning \& Innovation. Cambridge (UK): Cambridge University Press, 2000.

KIM, L. Imitation to Innovation - The Dynamics of Korea's Technological Learning. Cambridge (MA): Harvard Business School Press, 1997.

KIM, L. Building technological capability for industrialization: analytical frameworks and Korea's experience. Industrial and Corporate Change, v. 8 , n. 1, 1999.

KREMER, M. Creating Markets for N ew Vaccines. Cambridge (MA): Harvard University, The Brooking Institution, and NBER, 2000. 
LALL, S. Technological Changeand Industrialization in Asian N ewly Industrializing Economies. Cambridge (UK): Cambridge University Press, 2000.

LEE, K.; LIM , C. Technological regimes, catching-up and leapfrogging: findings from Korean industries. Research Policy, v. 30, n. 3, p. 459-483, 2001.

LOEWE, P; WILLIAMSON, P.; WOOD. Fivestyles of strategy innovation and how to use them. European Management Journal, v. 19, n. 2, p. 115-125, 2001.

MALERBA, F.; ORSENIGO, L. Technological regimes and firm behavior. Industrial and Corporate Change, p. 45-71, 1993.

MERRYLL LYNCH. Global vaccines. Biotehnology, jan/2003.

MYTELKA, L. K. Competition, innovation and competitiveness in developing countries. Competition, Innovation and Competitivenes. Paris: OECD Development Centre Studies, 1999.

NELSON, R.; WINTER, S. An Evolutionary Theory of Economic Change, Cambridge (MA): Harvard University Press, 1982.

NONAKA, I.; TAKEUCHI, H. TheKnowledge-Creating Company. N ew York: Oxford University Press, 1995.

NONAKA, I. et al. A firm as a knowledge-creating entity: a new perspective in the theory of the firm. Industrial and Corporate Change, mar/2000.

OECD. Biotechnology and Sustainability - The Fight against Infectious Disease, 2003.

PACK, $H$. Research and development in industrial development process. Technology, Learning \& Innovation. Cambridge (UK): Cambridge University Press, 2000.

PAVITT, K. What we know about the strategic management of technology. California Management Review, v. 32, n. 3, p. 17-26, 1990.
PENROSE, E. The Theory of Growth of theFirm. Oxford: Basil Blackwel, 1959.

PLOTKIN, S.; ORENSTEIN, W. Vaccines. 3. ed. Philadelphia: W. B. Saunders Company, 2000.

PORTER, M. Competitive Strategy. N ew York: The Free Press, 1980.

PORTER, M. Competitive Advantage. New York: The Free Press, 1985.

PRAHALAD, C.; HAMEL, G. The core competence of the corporation. Harvard Business Review, n. 3, p. 79-91, 1990.

PROENÇA, A. Dinâmica estratégica sob uma perspectiva analítica: refinando o entendimento gerencial. Rio de Janeiro: Arché, v. 8, n. 23, 1999.

TEECE, D. Profiting from technological innovation: implications for integration, collaboration, licensing and public policy. Research Policy, v. 15, p. 285-305, 1986.

TEECE, D.; PISANO G.; SHUEN, A. Dynamic capabilities and strategic management. Strategic M anagement Journal, v. 18, n. 7, p. 509-533, 1997.

TEMPORÃO, J. Complexo industrial da saúde: público e privado na produção e consumo de vacinas no Brasil. 2002. Tese de doutorado. Instituto de M edicina da UERJ - Rio de Janeiro.

TEMPORÃO, J. O Programa Nacional de Imunizações (PNI): origens e desenvolvimento. História, Ciências, Saúde- Manguinhos, v. 10, Suplemento 2, Rio de Janeiro, Fundação O swaldo Cruz, 2003.

THE JORDAN REPORT. Accelerated Development of Vaccines, US Department of Health and Human Services, 2002.

UTTERBACK, J. Mastering the Dynamics of Innovation. Cambridge (MA): Harvard Business School Press, 1994.

\section{Artigo recebido em 09.01.2004. Aprovado em 05.05.2004.}

\section{Rosiceli Barreto G onçalves Baetas}

Doutoranda em Gestão e Inovação Tecnológica na Escola de Química, UFRJ. Analista em C\& T Senior do Instituto de Tecnologia em Imunobiológicos, da F undação Oswaldo Cruz. Interesses de pesquisa na área de gestão e inovação tecnológica. E-mail: rosiceli@bio.fiocruz.br

Endereço: Av. Brasil, 4.365, Manguinhos, Pavilhão Rocha Lima, 60 andar, Rio de Janeiro-RJ, 21045-900.

\section{José Vítor Bomtempo}

Professor Adjunto da Escola de Química/UFRJ. DSc em Economia Industrial pela Ecole des M ines de Paris. Interesses de pesquisa na área de gestão e inovação tecnológica.

Email: vitor@eq.ufrj.br

Endereço: Escola de Química - UFRJ, Centro de Tecnologia, Bloco E, Fundão, Rio de Janeiro-RJ, 21949-900.

\section{C ristiane Quental}

Doutora em Administração de Empresas pela COPPEAD/UFRJ. Coordenadora de Estudos e Projetos Estratégicos da Diretoria de Planejamento Estratégico da Fundação Oswaldo Cruz. Coordenadora Adjunta do M estrado Profissional em Gestão de C\& T em Saúde, da Escola N acional de Saúde Pública/EN SP, da Fundação Oswal do Cruz. Interesses de pesquisa na área de planejamento estratégico.

E-mail: cquental@fiocruz.br

Endereço: Av. Brasil, 4.365, M anguinhos, Prédio Quinino, sala 305, Rio de Janeiro-RJ, 21045-900. 